\title{
MORE ON THE CONNECTIVITY OF CONVEX SETS
}

\author{
D. S. BRIDGES
}

\begin{abstract}
This note, written from a constructive viewpoint, shows how Mandelker's results on connectivity of compact intervals [3] extend to open balls in Banach space, and complete, convex subsets of arbitrary normed spaces. This is achieved by proving the equivalence of Mandelker's notion of connectivity with one of those discussed in [2].
\end{abstract}

1. As has been noted elsewhere [2], the search for a constructive theory of connectivity in metric spaces can be undertaken in at least two directions. One of these was followed by Mandelker in a paper [3] essentially restricted to connectivity of compact intervals. The other was taken in [2], which discusses connectivity for more general convex sets in normed spaces. In this note, we show how the work of both [2] and [3] enables us to extend Mandelker's results to sets of the latter type.

We recall that a subset $A$ of a metric space $E$ is located in $E$ if $\operatorname{dist}(x, A) \equiv$ $\inf \{d(x, y): y \in A\}$ is computable for each $x$ in $E$; in which case we can define the metric complement of $A$ in $E$ to be the set $E-A \equiv\{x \in E$ : $\operatorname{dist}(x, A)>0\}$.

We define a metric space $E$ to be

$O$-connected if, when $A$ is an open, located subset of $E$ with nonvoid metric complement, there exists $\xi$ in $\bar{A}$ such that $d(\xi, x)>0$ for each $x$ in $A$;

$M$-connected if, when $U, V$ are nonvoid, disjoint, open subsets of $E$, at least one of which is located, there exists $\xi$ in $E$ such that $d(\xi, x)>0$ for each $x$ in $U \cup V$.

Proposition 1. A metric space $E$ is O-connected if and only if it is M-connected.

Proof. If $E$ is $O$-connected and $U, V$ are disjoint, nonvoid, open subsets of $E$ with $U$ located, let $v$ be any point of $V$ and choose $r>0$ so that $B(0, r) \subset V$. Then $\operatorname{dist}(v, U) \geqslant r>0$, and $v \in E-U$. Thus there exists $\xi$ in $\bar{U}$ with $d(\xi, x)>0$ for each $x$ in $U$. As we also have $d(\xi, v) \geqslant r>0$, we see that $E$ is $M$-connected. Conversely, suppose that $E$ is $M$-connected, and let $A \subset E$ be open and located, with nonvoid metric complement. Then, as $E-A$ is open, there exists $\zeta$ in $E$ with $d(\zeta, x)>0$ for each $x$ in $A \cup(E-$ $A)$. Were $\operatorname{dist}(\zeta, A)>0$, we would have the contradiction $\zeta \in(E-A)$.

Received by the editors May 18, 1977.

AMS (MOS) subject classifications (1970). Primary 02E99, 46B05.

Key words and phrases. Convex, connected, constructive. 
Hence $\operatorname{dist}(\zeta, A)=0, \zeta \in \bar{A}$, and $E$ is $O$-connected.

Taken with 2.2 and 2.4 of [2], this immediately yields

THEOREM 1. If $S$ is either an open ball in a Banach space, or a complete, convex set in a normed space, then $S$ is $M$-connected.

This last result both subsumes Mandelker's Theorem 1 and weakens his hypotheses.

For a substantially complete picture of the relation between connected subsets of the real line and intervals, we may now refer to $\$ 3$ of [2]: in view of Proposition 1 above and 2.1 of [2], the results of that section remain valid with the words ' $O$-connected' and 'connected' replaced throughout by ' $M$ connected'.

2. It is natural to seek analogues of Mandelker's Theorems with open sets replaced by closed ones. In order to do so, let us call two subsets $U, V$ of a metric space strongly disjoint if $d(u, v)>0$ for each $u$ in $U$ and $v$ in $V$.

THEOREM 2. Let $S$ be either an open ball in a Banach space, or a complete, convex set in a normed space. Let $U, V$ be subsets of $S$ that are closed and located in $S$, and strongly disjoint. Then there exists a point $\xi$ of $S$ at positive distance from both $U$ and $V$.

Proof. We prove only the harder case, where $S$ is an open ball in a Banach space. Let $v \in V$ and choose $r>0$ so that $B(v, r) \subset S$. For each $x$ in $\bar{U}$, we have either $0<\|v-x\|$ or $\|v-x\|<r$; in the latter case, $x \in \bar{U} \cap S=$ $U$, and so $0<\|v-x\|$. As $\bar{U}$ is a complete, located subset of $\bar{S}$, $\operatorname{dist}(v, \bar{U})=$ $\operatorname{dist}(v, U)>0$ [1, Chapter 6, Lemma 7]. Thus $v \in S-U$. Applying 2.2 of [2], we now can construct $\zeta \in U \cap(S-U)^{-}$. An argument similar to the above then shows that $\delta \equiv \operatorname{dist}(\zeta, V)>0$. To complete the proof, it suffices to choose $\xi$ in $S-U$ with $\| \xi-\zeta \rrbracket<\delta$.

Note that both the hypotheses and the conclusion of Theorem 2 are stronger than those of Theorem 1. However, if the same strengthening of hypotheses is made in the latter, we do not arrive at a correspondingly stronger conclusion: to see this, just take $S=[0,1], U=\left[0, \frac{1}{2}[\right.$ and $V=$ ]$\left.\frac{1}{2}, 1\right]$.

3. Finally, we observe that, as an examination of their proofs readily shows, Mandelker's Theorems 2 and 3 generalise as follows:

THEOREM 3. If a convex set in a normed space is the union of two nonvoid, open (resp. closed) subsets, then these subsets have a common point.

THEOREM 4. If a convex set $S$ in a normed space is the union of two disjoint open (resp. closed) subsets, then one of these subsets is $S$, and the other is empty. 


\section{REFERENCES}

1. E. Bishop, Foundations of constructive analysis, McGraw-Hill, New York, 1967.

2. D. S. Bridges, On the connectivity of convex sets, Bull. London Math. Soc. 10 (1977).

3. M. Mandelker, Connectivity of an interval, Proc. Amer. Math. Soc. 54 (1976), 170-172.

Department of Mathematics, The University College at Buckingham, Hunter Street, BUCKINGHAM, MK 18 IEG, ENGLAND 